\title{
Comparative Exposure Assessment Using Silicone Passive Samplers Indicates Domestic Dogs are Sentinels to Support Human Health Research
}

Catherine F. Wise, ${ }^{\dagger,}$ Stephanie C. Hammel, ${ }^{\S}$ Nicholas Herkert, ${ }^{\S}$ Jun Ma,,$\stackrel{\perp}{ }$ Alison MotsingerReif, ${ }^{\#}$ Heather M. Stapleton, $\$, \nabla$ and Matthew Breen* $\uparrow, \downarrow, \nabla, \circ$,

†Department of Biological Sciences, Environmental and Molecular Toxicology Program, North Carolina State University, 850 Main Campus Drive, Raleigh, North Carolina 27606, United States.

†Department of Molecular Biomedical Sciences, College of Veterinary Medicine, North Carolina State University, 1060 William Moore Drive, Raleigh, North Carolina 27607, United States. $\$ N i c h o l a s$ School of the Environment, Duke University, Durham, North Carolina 27708, United States.

"Department of Statistics, North Carolina State University, Raleigh, North Carlina 27607, United States.

${ }^{\perp}$ Bioinformatics Research Center, North Carolina State University, Raleigh, North Carolina 27607, United States.

\#Biostatistics and Computational Biology Branch, National Institute of Environmental Health Sciences, Durham, North Carolina 27709, United States.

${ }^{\nabla}$ Duke Cancer Institute, Durham, North Carolina, United States.

$21{ }^{\circ}$ Comparative Medicine Institute, North Carolina State University, Raleigh, North Carolina

22 27607, United States. North Carolina 27607, United States.

*Matthew Breen PhD CBiol FRSB

Oscar J. Fletcher Distinguished Professor of Comparative Oncology Genetics

Dept. of Molecular Biomedical Sciences

North Carolina State University

College of Veterinary Medicine

CVM Research Building - Room 348

1060 William Moore Drive

Raleigh, North Carolina 27607 USA

Ph. 919-513-1467

Fax. 919-513-7301

E-mail Matthew_Breen@ncsu.edu

Lab site: http://www.BreenLab.org 


\section{Contents}

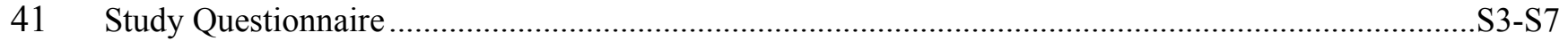

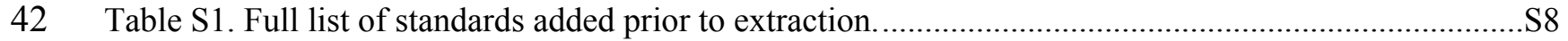

43 Table S2. Complete List of Standards added after sample extraction to calculate recovery of the internal

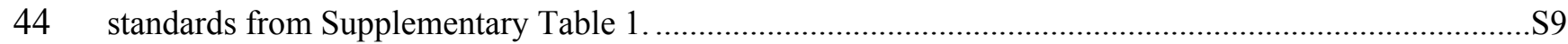

45 Table S3. Linear regression results for human wristband and dog tag................................................S10

46 Table S4. Linear regression results for $\operatorname{dog}$ tag and dog urine. ............................................................ 11

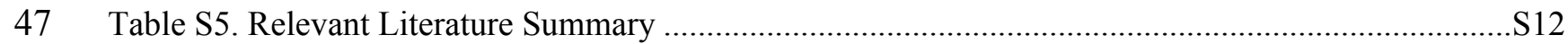

48 Figure S1. Spearman correlation coefficient heatmap grouped by chemical class. ...............................S15

49 Figure S2. Heatmap of Spearman's correlation coefficients for each chemical class. .....................S16-S20

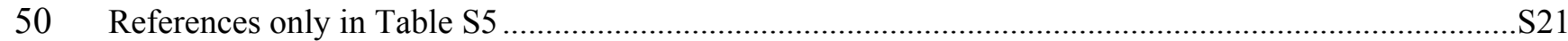




\section{Study Questionnaire}

Thank you for your participation in our study. Please complete the following questions as honestly as possible. All of your responses will be kept strictly confidential.

\section{OWNER}

1. Name:

2. Current Address:

3. Year of Birth

Month of Birth

4. What is your gender?
$\square$ Male
$\square$ Female

5. Do you smoke cigarettes?
$\square$ Yes, currently
- If so, for how many years?
No
- How many per day?

6. How many years have you lived in your current home?

$<6$ months
6-11 months
1-2 years
3-4 years
5-6 years
7+ years

7. How long ago was your home built?

$<12$ months ago
$1-5$ years ago
$6-10$ years ago
$11-20$ years ago
$21-50$ years ago
$>50$ years ago

8. Which description best fits your home? Apartment/Condo Townhouse Detached Single Home

9. On average, how many hours a day do you spend in your home?

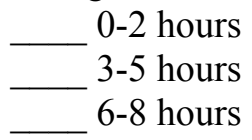


9-12 hours 13-18 hours 19-22 hours 24 hours

10. Of the hours a day spent in your home, please rank the following rooms as 1 (spend the most time in) to 5 (spend the least time in): Bedroom

Kitchen Family room/sitting room Computer room Garage/outdoors Other, Please specify:

\section{DOG}

1. Please indicate the Breed of your dog:

2. AKC Registration \# (if applicable):

3. What is your dog's weight?

4. Current age of dog (years):

5. Age at spay/neuter (if applicable)

6. What is the sex of your dog? Intact Female Intact Male Spayed Female Neutered Male

7. How long has your dog lived in your current home? $<6$ months 6-11 months $1-3$ years 4-8 years 9-12 years $12+$ years

8. How many hours per day does your dog spend inside your home on average?

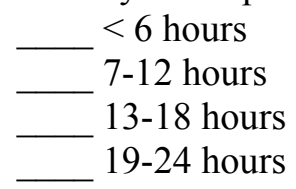

9. What is the average amount of exercise your dog gets per week? None or scarce activity (0-1 hour of walk per week) Moderate outdoor activity (1-2 hours) Regular outdoor activity (2-5 hours) Intensive outdoor activity ( $>5$ hours, including hunting) 
10. How many times a day does your dog urinate on average?

$1-3$ times
$4-6$ times
$7-9$ times
$>10$ times

11. Have you recently noticed your dog struggling to urinate?

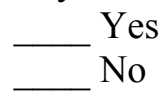

12. Have you recently noticed any change in the color of your dog's urine?

Yes
No
Unknown

13. Besides overnight, what is the longest amount of time your dog is required to hold their urine?

$<1$ hour
$>>1$ hour but $<3$ hours
$>$
$>$

14. What is your dog's main diet? (please check all that apply)

Dry food
Wet food
Raw
Vegetables
Cooked
Human food

15. On average, what type of water does your dog consume most frequently (more than $75 \%$ of the time)?

\begin{tabular}{ll} 
& Municipal water \\
& Private well water \\
& Community well water \\
& Bottled water \\
\hline & Filtered water \\
& Combination of tap, bottled, and/or filtered \\
& Other, please specify__
\end{tabular}

16. Does your dog regularly drink water from other sources such as ponds, streams, toilet bowls etc.?

Yes

- If Yes, please specify source and frequency

17. Do you routinely apply flea or tick medication to your dog?

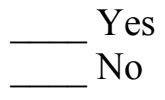

18. Did you apply a flea and tick medication to your dog while during the 5 day study period? Yes, topical ointment applied to skin 

Yes, currently wears a flea/tick collar Yes, oral medication No, Please list date of most recent application

19. Please list all the flea and tick medications given to your dog over the last year and their frequency.

20. Please list all the herbicides and pesticides used in your yard over the last year and their frequency (e.g., Roundup or 2,4-D). If applicable, please list lawn service used and frequency.

21. Please list all the pesticides used INSIDE your home over the last year and their frequency (e.g., Raid or Ortho).

22. When is your dog permitted access to the lawn following herbicide and/or pesticide treatment? Immediately

__ Less than 24 hours

24-48 hours

Greater than 48 hours

N_ Not applicable

23. Has your dog ever had any urinary tract issues (e.g. cystitis)?

$\underset{\text { No }}{\mathrm{Nes}}$

24. Has your dog ever been diagnosed with cancer?

$$
\begin{aligned}
& \text { Yes } \\
& \text { No } \\
& \text { - If yes, what type of cancer: } \\
& \text { - In what year was the cancer diagnosed: }
\end{aligned}
$$

25. Is your dog currently on any medications, NSAIDS, antibiotics, chemotherapy?

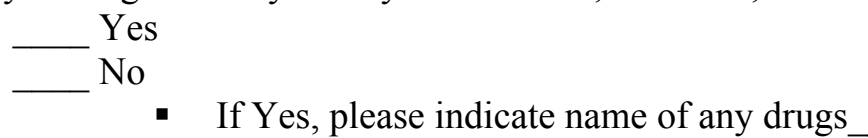


26. Does your dog have any other health related issues?

27. Where did your dog come from? Animal Shelter Pet Store Breeder Other, please indicate

28. Is your dog exposed to passive tobacco smoke?

Y

29. Does your dog receive any professional grooming?

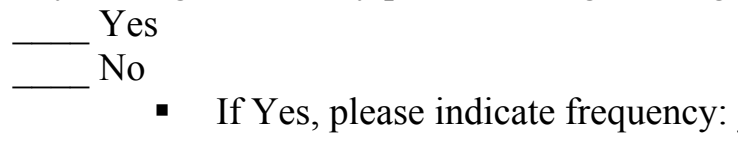

30. Does your dog have any in home personal care products, such as perfumes or shampoos?

Yes

31. Do you have, or have you had, additional dogs in your home? Yes No

- If YES, do any of the other dogs have bladder/urination issues?

Yes
No
Unknown


Table S1. Full list of standards added prior to extraction.

\begin{tabular}{|c|c|c|c|c|}
\hline Compound & Vendor & Catalog Number & Concentration (ng/mL) & Recovery $\% \pm$ SD \\
\hline d10- chlorpyrifos & Cambridge & DLM-4360-1.2 & 2000 & $54 \pm 11$ \\
\hline $13 \mathrm{C}$ cis permethrin & Cambridge & CLM-7322-1.2 & 1000 & $82 \pm 4$ \\
\hline dTBP & Wellington & dTBP & 1000 & $83 \pm 14$ \\
\hline 13C TBEP & Wellington & M6TBEP & 1000 & $7 \pm 5$ \\
\hline 13C TPP & Wellington & MTPP & 1000 & $92 \pm 8$ \\
\hline dTCEP & Wellington & dTCEP & 1000 & $74 \pm 18$ \\
\hline dTDCPP & Wellington & dTDCPP & 1000 & $90 \pm 9$ \\
\hline FBDE69 & Accustandard & $4001 \mathrm{~S}$ & 1000 & $96 \pm 12$ \\
\hline $13 \mathrm{C}$ TBB & Wellington & MEHTBB & 1000 & $67 \pm 17$ \\
\hline 13C TBPH & Wellington & MBEHTBP & 1000 & $103 \pm 28$ \\
\hline 13C BDE209 & Wellington & MBDE-209 & 500 & $49 \pm 8$ \\
\hline 13С РСB 52 & Wellington & MBP-52 & 1000 & $68 \pm 6$ \\
\hline 13C РCB 153 & Wellington & MBP-153 & 1000 & $79 \pm 4$ \\
\hline dDMP & Cambridge & DLM-1366-1.2 & 2000 & $56 \pm 13$ \\
\hline dDEP & Cambridge & DLM-1629-1.2 & 2000 & $65 \pm 10$ \\
\hline $\mathrm{dBBP}$ & Cambridge & DLM-1369-1.2 & 2000 & $82 \pm 6$ \\
\hline dDEHP & Cambridge & DLM-1368-1.2 & 2000 & $85 \pm 11$ \\
\hline
\end{tabular}


283 Table S2. Complete List of Standards added after sample extraction to calculate recovery 284 of the internal standards from Supplementary Table 1.

\begin{tabular}{|l|l|l|l|}
\hline Compound & Vendor & Catalog Number & Mass Added \\
\hline 13C CDE141 & Wellington & MCDE-141 & 1000 \\
\hline FBDE208 & Accustandard & FBDE-9001S & 1000 \\
\hline $13 C$ DCPH & Cambridge & CLM-4670-1.2 & 2000 \\
\hline dTCPP & Cambridge & DLM-9317-1.2 & 2000 \\
\hline dTPP & Wellington & dTPP & 1000 \\
\hline M2 13C 8:2 FTOH & Wellington & M2FOET & 1000 \\
\hline
\end{tabular}


Table S3. Linear regression results for human wristband and dog tag.

287 The correlation of chemical concentrations between dog tag and human wristband

\begin{tabular}{|c|c|c|c|c|c|c|c|}
\hline Chemical group & Human Wristband & Dog Wristband & $\begin{array}{l}\text { Model's } \\
\text { p-value }\end{array}$ & $\begin{array}{l}\text { Intercept's } \\
\text { p-value }\end{array}$ & $\begin{array}{l}\text { Predictor } \\
\text { Variable' } \\
\text { s p-value }\end{array}$ & R-Squared & $\begin{array}{l}\text { Adjusted } \\
\text { R-Squared }\end{array}$ \\
\hline \multirow[t]{9}{*}{ Wristband_BFRs } & BDE 28,33 ng/g WB human & BDE 28,33 ng/g WB dog & 0.000260388 & 0.05423822 & 0.000260388 & 0.423600903 & 0.403015221 \\
\hline & BDE $47 \mathrm{ng} / \mathrm{g}$ WB human & BDE 47 ng/g WB dog & 0.001158973 & 0.000148251 & 0.001158973 & 0.339448139 & 0.315857001 \\
\hline & BDE $100 \mathrm{ng} / \mathrm{g}$ WB human & BDE $100 \mathrm{ng} / \mathrm{g}$ WB dog & 0.001158973 & 0.343026292 & 0.001158973 & 0.336878872 & 0.313195975 \\
\hline & BDE $154 \mathrm{ng} / \mathrm{g}$ WB human & BDE $154 \mathrm{ng} / \mathrm{g}$ WB dog & $1.39 \mathrm{E}-05$ & $1.12 \mathrm{E}-06$ & $1.39 \mathrm{E}-05$ & 0.567642729 & 0.552201397 \\
\hline & BDE $153 \mathrm{ng} / \mathrm{g}$ WB human & BDE $153 \mathrm{ng} / \mathrm{g}$ WB dog & 0.00018114 & $7.60 \mathrm{E}-05$ & 0.00018114 & 0.457953159 & 0.438594343 \\
\hline & BDE $138 \mathrm{ng} / \mathrm{g}$ WB human & BDE $138 \mathrm{ng} / \mathrm{g}$ WB dog & 0.009054197 & $2.19 \mathrm{E}-04$ & 0.009054197 & 0.231973105 & 0.204543573 \\
\hline & TBB ng/g WB human & TBB ng/g WB dog & 0.026213002 & $4.72 \mathrm{E}-11$ & 0.026213002 & 0.170591261 & 0.14096952 \\
\hline & TBPH WB human & TBPH ng/g WB dog & 0.00037664 & $4.72 \mathrm{E}-11$ & 0.000260388 & 0.41717773 & 0.396362649 \\
\hline & T4tBPPP $\mathrm{ng} / \mathrm{g}$ WB human & T4tBPPP ng/g WB dog & 0.020246144 & $3.23 \mathrm{E}-08$ & 0.020246144 & 0.304914027 & 0.280089528 \\
\hline \multirow[t]{2}{*}{ Wristband_PCBs } & PCB28 ng/g WB human & PCB28 ng/g WB dog & 0.00037664 & $6.99 \mathrm{E}-09$ & 0.00037664 & 0.368344573 & 0.345785451 \\
\hline & PCB101 ng/g WB human & PCB101 ng/g WB dog & $1.07 \mathrm{E}-05$ & $5.51 \mathrm{E}-11$ & $1.07 \mathrm{E}-05$ & 0.528609624 & 0.511774253 \\
\hline \multirow[t]{3}{*}{ Wristband_Phthalates } & DMP ng/g WB human & DMP ng/g WB dog & $4.49 \mathrm{E}-05$ & $5.45 \mathrm{E}-22$ & $4.49 \mathrm{E}-05$ & 0.530879448 & 0.514125143 \\
\hline & DiBP ng/g WB human & DiBP ng/g WB dog & 0.024198856 & $2.85 \mathrm{E}-17$ & 0.024198856 & 0.2454058 & 0.218456008 \\
\hline & TOTM ng/g WB human & TOTM ng/g WB dog & 0.024844821 & $3.80 \mathrm{E}-19$ & 0.024844821 & 0.223820667 & 0.196099977 \\
\hline \multirow[t]{5}{*}{ Wristband_Pesticides } & Chlorpyrifos ng/g WB human & Chlorpyrifos ng/g WB dog & $1.85 \mathrm{E}-05$ & $8.77 \mathrm{E}-05$ & $1.85 \mathrm{E}-05$ & 0.546389084 & 0.530188695 \\
\hline & trans-Chlordane ng/g WB human & trans-Chlordane ng/g WB dog & $4.34 \mathrm{E}-05$ & 0.018230175 & $4.34 \mathrm{E}-05$ & 0.480585087 & 0.462034554 \\
\hline & cis-Chlordane ng/g WB human & cis-Chlordane ng/g WB dog & $2.12 \mathrm{E}-05$ & 0.083653689 & $2.12 \mathrm{E}-05$ & 0.51943565 & 0.502272637 \\
\hline & trans-Permethrin ng/g WB human & trans-Permethrin ng/g WB dog & 0.01346756 & $5.13 \mathrm{E}-11$ & 0.01346756 & 0.219737541 & 0.191871024 \\
\hline & cis-Permethrin ng/g WB human & cis-Permethrin ng/g WB dog & 0.040065068 & $1.35 \mathrm{E}-10$ & 0.040065068 & 0.151687539 & 0.121390665 \\
\hline \multirow[t]{12}{*}{ Wristband_OPEs } & TCIPP ng/g WB human & TCIPP ng/g WB dog & 0.029397785 & $6.79 \mathrm{E}-18$ & 0.029397785 & 0.174653353 & 0.145176687 \\
\hline & TPHP ng/g WB human & TPHP ng/g WB dog & 0.019206616 & $1.03 \mathrm{E}-15$ & 0.019206616 & 0.213045162 & 0.184939632 \\
\hline & 2IPPDPP ng/g WB human & 2IPPDPP ng/g WB dog & 0.008270627 & $8.12 \mathrm{E}-13$ & 0.008270627 & 0.333773022 & 0.309979201 \\
\hline & 3IPPDPP ng/g WB human & 3IPPDPP ng/g WB dog & 0.020520402 & 0.001055898 & 0.020520402 & 0.203648456 & 0.17520733 \\
\hline & B21PPPP ng/g WB human & B2IPPPP ng/g WB dog & 0.044003227 & $1.74 \mathrm{E}-10$ & 0.044003227 & 0.148893888 & 0.118497241 \\
\hline & 4IPPDPP ng/g WB human & 4IPPDPP ng/g WB dog & 0.008270627 & $1.25 \mathrm{E}-07$ & 0.008270627 & 0.302107985 & 0.27718327 \\
\hline & 24DIPPDPP ng/g WB human & 24DIPPDPP ng/g WB dog & 0.008755659 & $3.06 \mathrm{E}-10$ & 0.008755659 & 0.285882538 & 0.260378343 \\
\hline & 4tBPDPP ng/g WB human & 4tBPDPP ng/g WB dog & 0.014662609 & $3.35 \mathrm{E}-05$ & 0.014662609 & 0.234357948 & 0.207013589 \\
\hline & B31PPPP ng/g WB human & B3IPPPP ng/g WB dog & 0.022796447 & $1.72 \mathrm{E}-08$ & 0.022796447 & 0.192813934 & 0.16398586 \\
\hline & B4lPPPP ng/g WB human & B4IPPPP ng/g WB dog & 0.014662609 & 0.224640248 & 0.014662609 & 0.233413007 & 0.2060349 \\
\hline & B4tBPPP ng/g WB human & B4tBPPP ng/g WB dog & 0.0123126 & 0.392830338 & 0.0123126 & 0.258697878 & 0.232222803 \\
\hline & T4tBPPP $\mathrm{ng} / \mathrm{g}$ WB human & T4tBPPP ng/g WB dog & 0.008270627 & $7.69 \mathrm{E}-09$ & 0.008270627 & 0.304914027 & 0.280089528 \\
\hline
\end{tabular}

The FDR level is $0.05 . \mathrm{WB}=$ wristband 
Table S4. Linear regression results for dog tag and dog urine.

290 The correlation of chemical concentrations between dog tag and dog urine (OPEs only)

\begin{tabular}{|l|l|l|l|l|l|l|l|}
\hline $\begin{array}{l}\text { Chemical } \\
\text { group }\end{array}$ & Dog Wristband & Dog Urine & $\begin{array}{l}\text { Model's } \\
\text { p-value }\end{array}$ & $\begin{array}{l}\text { Intercept's } \\
\mathbf{p} \text {-value }\end{array}$ & $\begin{array}{l}\text { Predictor } \\
\text { Variable's } \\
\text { p-value }\end{array}$ & R-Squared & $\begin{array}{l}\text { Adjusted } \\
\text { R-Squared }\end{array}$ \\
\hline $\begin{array}{l}\text { Urine OPE } \\
\text { metabolite } \\
\text { raw data }\end{array}$ & TDCIPP ng/g WB & $\begin{array}{l}\text { BDCIPP ng/ml } \\
\text { raw value }\end{array}$ & 0.02857 & 0.23416 & 0.02857 & 0.18111 & 0.15187 \\
\hline & TPHP ng/g WB & $\begin{array}{l}\text { DPHP ng/ml } \\
\text { raw value }\end{array}$ & 0.00350 & 0.17472 & 0.00350 & 0.31823 & 0.29388 \\
\hline $\begin{array}{l}\text { Urine OPE } \\
\text { metabolite }\end{array}$ & TDCIPP ng/g WB & $\begin{array}{l}\text { BDCIPP ng/ml } \\
\text { SG corrected }\end{array}$ & 0.04221 & 0.09496 & 0.04221 & 0.16070 & 0.13072 \\
\hline & TPHP ng/g WB & $\begin{array}{l}\text { DPHP ng/ml } \\
\text { SG corrected }\end{array}$ & 0.00109 & 0.02345 & 0.00109 & 0.36972 & 0.34721 \\
\hline
\end{tabular}

291 The FDR level is $0.05 . \mathrm{WB}=$ wristband; $\mathrm{SG}=$ specific gravity 
292 Table S5. Relevant Literature Summary

\begin{tabular}{|c|c|c|c|c|c|c|c|c|c|}
\hline Chemical & $\begin{array}{l}\text { DF } \\
\%\end{array}$ & $\begin{array}{c}\text { Total } \\
\mathbf{N}\end{array}$ & $\begin{array}{l}\text { Statistic } \\
\text { Type }\end{array}$ & Conc. & $\begin{array}{l}\text { Reported } \\
\text { Units }\end{array}$ & $\begin{array}{l}\text { Days } \\
\text { worn }\end{array}$ & $\begin{array}{c}\text { Population } \\
\text { Type }\end{array}$ & Location & Reference \\
\hline \multicolumn{10}{|l|}{ OPEs } \\
\hline \multirow[t]{6}{*}{ TCEP } & 100 & 10 & Median & 60.9 & $\mathrm{ng} / \mathrm{g}$ & 7 & $\begin{array}{l}\text { General } \\
\text { population }\end{array}$ & Indiana, USA & 24 \\
\hline & 89 & 72 & N.R. & N.R. & ng/g/day & 7 & Children & Oregon, USA & 23 \\
\hline & 89 & 38 & Median & 108.5 & $\mathrm{ng} / \mathrm{g}$ & 7 & Mothers & New York, USA & 20 \\
\hline & 76 & 38 & Median & 64.5 & $\mathrm{ng} / \mathrm{g}$ & 7 & Children & New York, USA & 20 \\
\hline & 51.3 & 39 & GM & 56.4 & $\mathrm{pmol} / \mathrm{g}$ & 7 & $\begin{array}{l}\text { Hyperthyroid } \\
\text { cats }\end{array}$ & $\begin{array}{l}\text { New York \& Oregon, } \\
\text { USA }\end{array}$ & 27 \\
\hline & 56.4 & 39 & GM & 76 & $\mathrm{pmol} / \mathrm{g}$ & 7 & $\begin{array}{l}\text { Nonhyperthyroid } \\
\text { cats }\end{array}$ & $\begin{array}{l}\text { New York \& Oregon, } \\
\text { USA }\end{array}$ & 27 \\
\hline \multirow[t]{7}{*}{ TDCIPP } & 100 & 10 & Median & 759 & $\mathrm{ng} / \mathrm{g}$ & 7 & $\begin{array}{l}\text { General } \\
\text { population }\end{array}$ & Indiana, USA & 24 \\
\hline & 96 & 72 & N.R. & N.R. & ng/g/day & 7 & Children & Oregon, USA & 23 \\
\hline & 100 & 40 & GM & 1251 & ng/band & 5 & $\begin{array}{l}\text { General } \\
\text { population }\end{array}$ & North Carolina, USA & 7 \\
\hline & 97 & 38 & Median & 163.5 & $\mathrm{ng} / \mathrm{g}$ & 7 & Mothers & New York, USA & 20 \\
\hline & 97 & 38 & Median & 390.5 & $\mathrm{ng} / \mathrm{g}$ & 7 & Children & New York, USA & 20 \\
\hline & 94.9 & 39 & GM & 146 & $\mathrm{pmol} / \mathrm{g}$ & 7 & $\begin{array}{l}\text { Hyperthyroid } \\
\text { cats }\end{array}$ & $\begin{array}{l}\text { New York \& Oregon, } \\
\text { USA }\end{array}$ & 27 \\
\hline & 97.4 & 39 & GM & 126 & $\mathrm{pmol} / \mathrm{g}$ & 7 & $\begin{array}{l}\text { Nonhyperthyroid } \\
\text { cats }\end{array}$ & $\begin{array}{l}\text { New York \& Oregon, } \\
\text { USA }\end{array}$ & 27 \\
\hline \multirow[t]{7}{*}{ TCIPP } & 100 & 10 & Median & 288 & $\mathrm{ng} / \mathrm{g}$ & 7 & $\begin{array}{l}\text { General } \\
\text { population }\end{array}$ & Indiana, USA & 24 \\
\hline & 92 & 72 & N.R. & N.R. & ng/g/day & 7 & Children & Oregon, USA & 23 \\
\hline & 100 & 40 & GM & 1536 & ng/band & 5 & $\begin{array}{l}\text { General } \\
\text { population }\end{array}$ & North Carolina, USA & 7 \\
\hline & 45 & 38 & Median & 4.2 & $\mathrm{ng} / \mathrm{g}$ & 7 & Mothers & New York, USA & 20 \\
\hline & 74 & 38 & Median & 297.5 & $\mathrm{ng} / \mathrm{g}$ & 7 & Children & New York, USA & 20 \\
\hline & 100 & 39 & GM & 2070 & $\mathrm{pmol} / \mathrm{g}$ & 7 & $\begin{array}{l}\text { Hyperthyroid } \\
\text { cats }\end{array}$ & $\begin{array}{l}\text { New York \& Oregon, } \\
\text { USA }\end{array}$ & 27 \\
\hline & 94.9 & 39 & GM & 1860 & $\mathrm{pmol} / \mathrm{g}$ & 7 & $\begin{array}{l}\text { Nonhyperthyroid } \\
\text { cats }\end{array}$ & $\begin{array}{l}\text { New York \& Oregon, } \\
\text { USA }\end{array}$ & 27 \\
\hline TBoEP & 100 & 10 & Median & 2090 & $\mathrm{ng} / \mathrm{g}$ & 7 & $\begin{array}{l}\text { General } \\
\text { population }\end{array}$ & Indiana, USA & 24 \\
\hline \multirow[t]{7}{*}{ TPHP } & 100 & 10 & Median & 290 & $\mathrm{ng} / \mathrm{g}$ & 7 & $\begin{array}{l}\text { General } \\
\text { population }\end{array}$ & Indiana, USA & 24 \\
\hline & 97 & 72 & Median & N.R. & ng/g/day & 7 & Children & Oregon, USA & 23 \\
\hline & 100 & 40 & GM & 459.5 & ng/band & 5 & $\begin{array}{l}\text { General } \\
\text { population }\end{array}$ & North Carolina, USA & 7 \\
\hline & 100 & 38 & Median & 399 & $\mathrm{ng} / \mathrm{g}$ & 7 & Mothers & New York, USA & 20 \\
\hline & 100 & 38 & Median & 440 & $\mathrm{ng} / \mathrm{g}$ & 7 & Children & New York, USA & 20 \\
\hline & 94.9 & 39 & GM & 164 & $\mathrm{pmol} / \mathrm{g}$ & 7 & $\begin{array}{l}\text { Hyperthyroid } \\
\text { cats }\end{array}$ & $\begin{array}{l}\text { New York \& Oregon, } \\
\text { USA }\end{array}$ & 27 \\
\hline & 100 & 39 & GM & 256 & $\mathrm{pmol} / \mathrm{g}$ & 7 & $\begin{array}{l}\text { Nonhyperthyroid } \\
\text { cats }\end{array}$ & $\begin{array}{l}\text { New York \& Oregon, } \\
\text { USA }\end{array}$ & 27 \\
\hline EHDPP & 85 & 10 & Median & 245 & $\mathrm{ng} / \mathrm{g}$ & 7 & $\begin{array}{l}\text { General } \\
\text { population }\end{array}$ & Indiana, USA & 24 \\
\hline \multicolumn{10}{|l|}{ BFRs } \\
\hline \multirow[t]{4}{*}{ BDE-28,33 } & 100 & 30 & GM & 2.2 & $\mathrm{ng} / \mathrm{g}$ & 7 & $\begin{array}{l}\text { General } \\
\text { population }\end{array}$ & North Carolina, USA & 5 \\
\hline & 61 & 72 & N.R. & N.R. & ng/g/day & 7 & Children & Oregon, USA & 23 \\
\hline & 5.1 & 39 & GM & $<$ LOD & $\mathrm{pmol} / \mathrm{g}$ & 7 & $\begin{array}{l}\text { Hyperthyroid } \\
\text { cats }\end{array}$ & $\begin{array}{l}\text { New York \& Oregon, } \\
\text { USA }\end{array}$ & 27 \\
\hline & 10.3 & 39 & GM & $<$ LOD & $\mathrm{pmol} / \mathrm{g}$ & 7 & $\begin{array}{l}\text { Nonhyperthyroid } \\
\text { cats }\end{array}$ & $\begin{array}{l}\text { New York \& Oregon, } \\
\text { USA }\end{array}$ & 27 \\
\hline \multirow[t]{5}{*}{ BDE-47 } & 100 & 10 & GM & 6.21 & $\mathrm{ng} / \mathrm{g}$ & 7 & $\begin{array}{l}\text { General } \\
\text { population }\end{array}$ & Indiana, USA & 24 \\
\hline & 96 & 72 & N.R. & N.R. & ng/g/day & 7 & Children & Oregon, USA & 23 \\
\hline & 100 & 30 & GM & 55.9 & $\mathrm{ng} / \mathrm{g}$ & 7 & $\begin{array}{l}\text { General } \\
\text { population }\end{array}$ & North Carolina, USA & 5 \\
\hline & 89.7 & 39 & GM & 24.5 & $\mathrm{pmol} / \mathrm{g}$ & 7 & $\begin{array}{l}\text { Hyperthyroid } \\
\text { cats }\end{array}$ & $\begin{array}{l}\text { New York \& Oregon, } \\
\text { USA }\end{array}$ & 27 \\
\hline & 84.6 & 39 & GM & 24.8 & $\mathrm{pmol} / \mathrm{g}$ & 7 & $\begin{array}{l}\text { Nonhyperthyroid } \\
\text { cats }\end{array}$ & $\begin{array}{l}\text { New York \& Oregon, } \\
\text { USA }\end{array}$ & 27 \\
\hline
\end{tabular}




\begin{tabular}{|c|c|c|c|c|c|c|c|c|c|}
\hline BDE-100 & 90 & 72 & N.R. & N.R. & $\mathrm{ng} / \mathrm{g} / \mathrm{day}$ & 7 & Children & Oregon, USA & 23 \\
\hline & 100 & 10 & Median & 3.47 & $\mathrm{ng} / \mathrm{g}$ & 7 & $\begin{array}{l}\text { General } \\
\text { population }\end{array}$ & Indiana, USA & 24 \\
\hline & 100 & 30 & GM & 13.7 & $\mathrm{ng} / \mathrm{g}$ & 7 & $\begin{array}{l}\text { General } \\
\text { population }\end{array}$ & North Carolina, USA & 5 \\
\hline & 25.6 & 39 & GM & 2.45 & $\mathrm{pmol} / \mathrm{g}$ & 7 & $\begin{array}{l}\text { Hyperthyroid } \\
\text { cats }\end{array}$ & $\begin{array}{l}\text { New York \& Oregon, } \\
\text { USA }\end{array}$ & 27 \\
\hline & 35.9 & 39 & GM & 4.11 & $\mathrm{pmol} / \mathrm{g}$ & 7 & $\begin{array}{l}\text { Nonhyperthyroid } \\
\text { cats }\end{array}$ & $\begin{array}{l}\text { New York \& Oregon, } \\
\text { USA }\end{array}$ & 27 \\
\hline \multirow[t]{3}{*}{ BDE-138 } & 100 & 30 & GM & 0.34 & $\mathrm{ng} / \mathrm{g}$ & 7 & $\begin{array}{l}\text { General } \\
\text { population }\end{array}$ & North Carolina, USA & 5 \\
\hline & 0 & 39 & GM & $<$ LOD & $\mathrm{pmol} / \mathrm{g}$ & 7 & $\begin{array}{l}\text { Hyperthyroid } \\
\text { cats }\end{array}$ & $\begin{array}{l}\text { New York \& Oregon, } \\
\text { USA }\end{array}$ & 27 \\
\hline & 2.6 & 39 & GM & $<$ LOD & $\mathrm{pmol} / \mathrm{g}$ & 7 & $\begin{array}{l}\text { Nonhyperthyroid } \\
\text { cats }\end{array}$ & $\begin{array}{l}\text { New York \& Oregon, } \\
\text { USA }\end{array}$ & 27 \\
\hline \multirow[t]{5}{*}{ BDE-153 } & 100 & 10 & Median & 1.36 & $\mathrm{ng} / \mathrm{g}$ & 7 & $\begin{array}{l}\text { General } \\
\text { population }\end{array}$ & Indiana, USA & 24 \\
\hline & 90 & 72 & N.R. & N.R. & ng/g/day & 7 & Children & Oregon, USA & 23 \\
\hline & 100 & 30 & GM & 3.78 & $\mathrm{ng} / \mathrm{g}$ & 7 & $\begin{array}{l}\text { General } \\
\text { population }\end{array}$ & North Carolina, USA & 5 \\
\hline & 43.6 & 39 & GM & 1.56 & $\mathrm{pmol} / \mathrm{g}$ & 7 & $\begin{array}{l}\text { Hyperthyroid } \\
\text { cats }\end{array}$ & $\begin{array}{l}\text { New York \& Oregon, } \\
\text { USA }\end{array}$ & 27 \\
\hline & 46.2 & 39 & GM & 2.05 & $\mathrm{pmol} / \mathrm{g}$ & 7 & $\begin{array}{l}\text { Nonhyperthyroid } \\
\text { cats }\end{array}$ & $\begin{array}{l}\text { New York \& Oregon, } \\
\text { USA }\end{array}$ & 27 \\
\hline \multirow[t]{5}{*}{ BDE-154 } & 92 & 10 & Median & 1.03 & $\mathrm{ng} / \mathrm{g}$ & 7 & $\begin{array}{l}\text { General } \\
\text { population }\end{array}$ & Indiana, USA & 24 \\
\hline & 89 & 72 & N.R. & N.R. & ng/g/day & 7 & Children & Oregon, USA & 23 \\
\hline & 100 & 30 & GM & 3.26 & $\mathrm{ng} / \mathrm{g}$ & 7 & $\begin{array}{l}\text { General } \\
\text { population }\end{array}$ & North Carolina, USA & 5 \\
\hline & 23.1 & 39 & GM & $<$ LOD & $\mathrm{pmol} / \mathrm{g}$ & 7 & $\begin{array}{l}\text { Hyperthyroid } \\
\text { cats }\end{array}$ & $\begin{array}{l}\text { New York \& Oregon, } \\
\text { USA }\end{array}$ & 27 \\
\hline & 28.2 & 39 & GM & 1.14 & $\mathrm{pmol} / \mathrm{g}$ & 7 & $\begin{array}{l}\text { Nonhyperthyroid } \\
\text { cats }\end{array}$ & $\begin{array}{l}\text { New York \& Oregon, } \\
\text { USA }\end{array}$ & 27 \\
\hline BDE-181 & 50 & 30 & GM & 0.041 & $\mathrm{ng} / \mathrm{g}$ & 7 & $\begin{array}{l}\text { General } \\
\text { population }\end{array}$ & North Carolina, USA & 5 \\
\hline \multirow[t]{2}{*}{ BDE-183 } & 62 & 10 & Median & 0.28 & $\mathrm{ng} / \mathrm{g}$ & 7 & $\begin{array}{l}\text { General } \\
\text { population }\end{array}$ & Indiana, USA & 24 \\
\hline & 100 & 30 & GM & 0.46 & $\mathrm{ng} / \mathrm{g}$ & 7 & $\begin{array}{l}\text { General } \\
\text { population }\end{array}$ & North Carolina, USA & 5 \\
\hline \multirow[t]{2}{*}{ BDE-209 } & 50 & 30 & GM & 12.2 & $\mathrm{ng} / \mathrm{g}$ & 7 & $\begin{array}{l}\text { General } \\
\text { population }\end{array}$ & North Carolina, USA & 5 \\
\hline & 62 & 10 & GM & 3.71 & $\mathrm{ng} / \mathrm{g}$ & 7 & $\begin{array}{l}\text { General } \\
\text { population }\end{array}$ & Indiana, USA & 24 \\
\hline \multirow[t]{3}{*}{ EH-TBB } & 100 & 30 & GM & 43 & $\mathrm{ng} / \mathrm{g}$ & 7 & $\begin{array}{l}\text { General } \\
\text { population }\end{array}$ & North Carolina, USA & 5 \\
\hline & 7.7 & 39 & GM & $<$ LOD & $\mathrm{pmol} / \mathrm{g}$ & 7 & $\begin{array}{l}\text { Hyperthyroid } \\
\text { cats }\end{array}$ & $\begin{array}{l}\text { New York \& Oregon, } \\
\text { USA }\end{array}$ & 27 \\
\hline & 7.9 & 39 & GM & 9.39 & $\mathrm{pmol} / \mathrm{g}$ & 7 & $\begin{array}{l}\text { Nonhyperthyroid } \\
\text { cats }\end{array}$ & $\begin{array}{l}\text { New York \& Oregon, } \\
\text { USA }\end{array}$ & 27 \\
\hline BEH-EBP & 100 & 10 & GM & 32.6 & $\mathrm{ng} / \mathrm{g}$ & 7 & $\begin{array}{l}\text { General } \\
\text { population }\end{array}$ & Indiana, USA & 24 \\
\hline \multicolumn{10}{|l|}{ PCBs } \\
\hline PCB & N.R. & 68 & N.R. & N.R. & & $\begin{array}{l}30- \\
34\end{array}$ & $\begin{array}{l}\text { General } \\
\text { population }\end{array}$ & $\begin{array}{l}\text { Alto Mayo region, } \\
\text { Peru }\end{array}$ & 56 \\
\hline \multicolumn{10}{|l|}{ Pesticides } \\
\hline Lindane & $20^{*}$ & 35 & N.R. & N.R. & $\mathrm{ng} / \mathrm{g}$ & $5 \times 2$ & Farming Families & Diender, Senegal & 57 \\
\hline \multirow[t]{4}{*}{ Chlorpyrifos } & 91 & 68 & Range & $\begin{array}{c}17- \\
1900\end{array}$ & $\mathrm{ng} / \mathrm{g}$ & $\begin{array}{l}30- \\
34\end{array}$ & $\begin{array}{l}\text { General } \\
\text { population }\end{array}$ & $\begin{array}{l}\text { Alto Mayo region, } \\
\text { Peru }\end{array}$ & 56 \\
\hline & $54^{*}$ & 35 & N.R. & N.R. & $\mathrm{ng} / \mathrm{g}$ & $5 \times 2$ & Farming Families & Diender, Senegal & 57 \\
\hline & 36.1 & 97 & Median & $<1.82$ & ng/g/day & 7 & $\begin{array}{l}\text { Female } \\
\text { Teenager }\end{array}$ & California, USA & 37 \\
\hline & 50 & 10 & Range & $\begin{array}{c}<0.444- \\
202\end{array}$ & $\mathrm{ng} / \mathrm{g}$ & $7-14$ & $\begin{array}{l}\text { Farmworker } \\
\text { Children }\end{array}$ & North Carolina, USA & 58 \\
\hline $\begin{array}{c}\text { Trans- } \\
\text { Chlordane }\end{array}$ & 70 & 10 & Range & $\begin{array}{c}<0.241- \\
16.7\end{array}$ & $\mathrm{ng} / \mathrm{g}$ & $7-14$ & $\begin{array}{l}\text { Farmworker } \\
\text { Children }\end{array}$ & North Carolina, USA & 58 \\
\hline $\begin{array}{c}\text { Cis- } \\
\text { Chlordane }\end{array}$ & $<50$ & 68 & N.R. & N.R. & $\mathrm{ng} / \mathrm{g}$ & $\begin{array}{l}30- \\
34\end{array}$ & $\begin{array}{l}\text { General } \\
\text { population }\end{array}$ & $\begin{array}{l}\text { Alto Mayo region, } \\
\text { Peru }\end{array}$ & 56 \\
\hline
\end{tabular}




\begin{tabular}{|c|c|c|c|c|c|c|c|c|c|}
\hline & 12.5 & 97 & N.R. & N.R. & ng/g/day & 7 & $\begin{array}{l}\text { Female } \\
\text { Teenager }\end{array}$ & California, USA & 37 \\
\hline & 80 & 10 & Range & $\begin{array}{c}<0.249- \\
34.6\end{array}$ & $\mathrm{ng} / \mathrm{g}$ & $7-14$ & $\begin{array}{l}\text { Farmworker } \\
\text { Children }\end{array}$ & North Carolina, USA & 58 \\
\hline \multirow[t]{2}{*}{$\begin{array}{c}\text { Trans- } \\
\text { Permethrin } \\
\end{array}$} & 51.5 & 97 & N.R. & N.R. & ng/g/day & 7 & $\begin{array}{l}\text { Female } \\
\text { Teenager }\end{array}$ & California, USA & 37 \\
\hline & 40 & 10 & Range & $\begin{array}{c}<0.310- \\
534\end{array}$ & $\mathrm{ng} / \mathrm{g}$ & $7-14$ & $\begin{array}{l}\text { Farmworker } \\
\text { Children }\end{array}$ & North Carolina, USA & 58 \\
\hline \multirow[t]{2}{*}{$\begin{array}{c}\text { Cis- } \\
\text { Permethrin }\end{array}$} & 48.5 & 97 & N.R. & N.R. & ng/g/day & 7 & $\begin{array}{l}\text { Female } \\
\text { Teenager }\end{array}$ & California, USA & 37 \\
\hline & 40 & 10 & Range & $\begin{array}{c}<0.178- \\
249\end{array}$ & $\mathrm{ng} / \mathrm{g}$ & $7-14$ & $\begin{array}{l}\text { Farmworker } \\
\text { Children }\end{array}$ & North Carolina, USA & 58 \\
\hline Permethrin & $<50$ & 68 & N.R. & N.R. & $\mathrm{ng} / \mathrm{g}$ & $\begin{array}{l}30- \\
34\end{array}$ & $\begin{array}{l}\text { General } \\
\text { population }\end{array}$ & $\begin{array}{l}\text { Alto Mayo region, } \\
\text { Peru }\end{array}$ & 56 \\
\hline Permethrin & $27^{*}$ & 35 & N.R. & N.R. & $\mathrm{ng} / \mathrm{g}$ & $5 \times 2$ & Farming Families & Diender, Senegal & 57 \\
\hline $\begin{array}{c}\Sigma \\
\text { Permethrin }\end{array}$ & 54.6 & 97 & median & 7.6 & ng/g/day & 7 & $\begin{array}{l}\text { Female } \\
\text { Teenager }\end{array}$ & California, USA & 37 \\
\hline \multirow[t]{4}{*}{ Cypermethrin } & 71 & 68 & Range & $\begin{array}{c}77- \\
7000\end{array}$ & $\mathrm{ng} / \mathrm{g}$ & $\begin{array}{l}30- \\
34\end{array}$ & $\begin{array}{l}\text { General } \\
\text { population }\end{array}$ & $\begin{array}{l}\text { Alto Mayo region, } \\
\text { Peru }\end{array}$ & 56 \\
\hline & 94 & 35 & N.R. & N.R. & $\mathrm{ng} / \mathrm{g}$ & $5 \times 2$ & Farming Families & Diender, Senegal & 57 \\
\hline & 55.7 & 97 & Median & 2.3 & ng/g/day & 7 & $\begin{array}{l}\text { Female } \\
\text { Teenager }\end{array}$ & California, USA & 37 \\
\hline & 40 & 10 & Range & $\begin{array}{l}<1.24- \\
212\end{array}$ & $\mathrm{ng} / \mathrm{g}$ & $7-14$ & $\begin{array}{l}\text { General } \\
\text { population }\end{array}$ & North Carolina, USA & 58 \\
\hline Azoxystrobin & 47 & 30 & Range & $0.1-13$ & $\mathrm{ng} / \mathrm{g}$ & 5 & $\begin{array}{l}\text { Farmworker } \\
\text { Children }\end{array}$ & Leuven, Belgium & 59 \\
\hline \multicolumn{10}{|l|}{ Phthalates } \\
\hline DMP & 100 & 27 & N.R. & N.R. & $\log n g / g$ & 5 & Teenagers & Santiago region, Chile & 60 \\
\hline DEP & 100 & 27 & N.R. & N.R. & $\log n g / g$ & 5 & Teenagers & Santiago region, Chile & 60 \\
\hline DBP & 100 & 27 & N.R. & N.R. & $\log n g / g$ & 5 & Teenagers & Santiago region, Chile & 60 \\
\hline BBP & 100 & 27 & N.R. & N.R. & $\log n g / g$ & 5 & Teenagers & Santiago region, Chile & 60 \\
\hline DEHP & 100 & 27 & N.R. & N.R. & $\log n g / g$ & 5 & Teenagers & Santiago region, Chile & 60 \\
\hline
\end{tabular}

293 N.R. not reported; GM geometric mean

$294 *$ Estimated frequencies of detection based on Donald et al 2016 Figure 1. 
Figure S1. Spearman correlation coefficient heatmap grouped by chemical class.

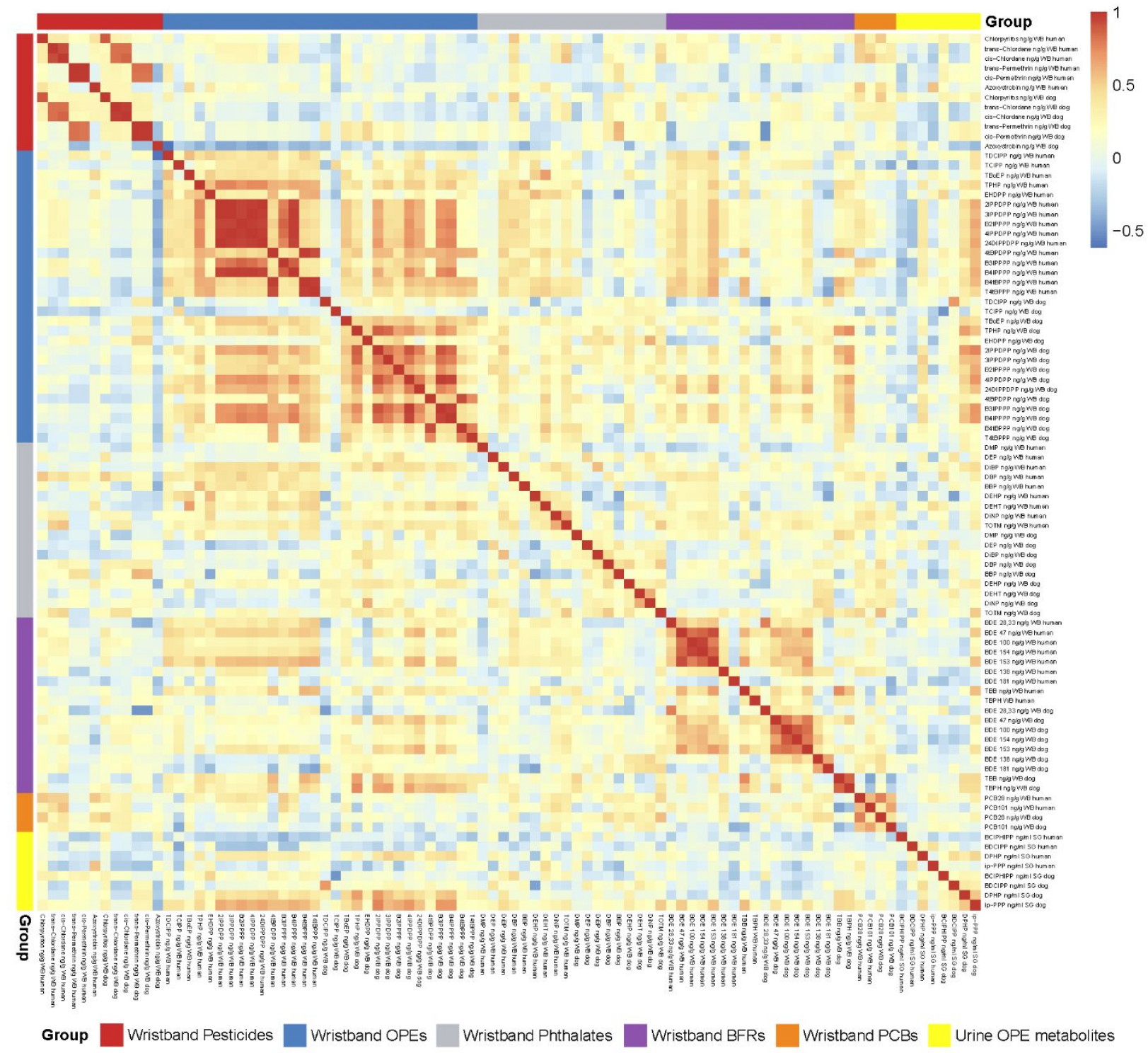


298 Pesticides (b) Organophosphate esters (OPEs) (c) Phthalates (d) Brominated flame retardants

299 (BFRs) (e) Polychlorinated biphenyls (PCBs)

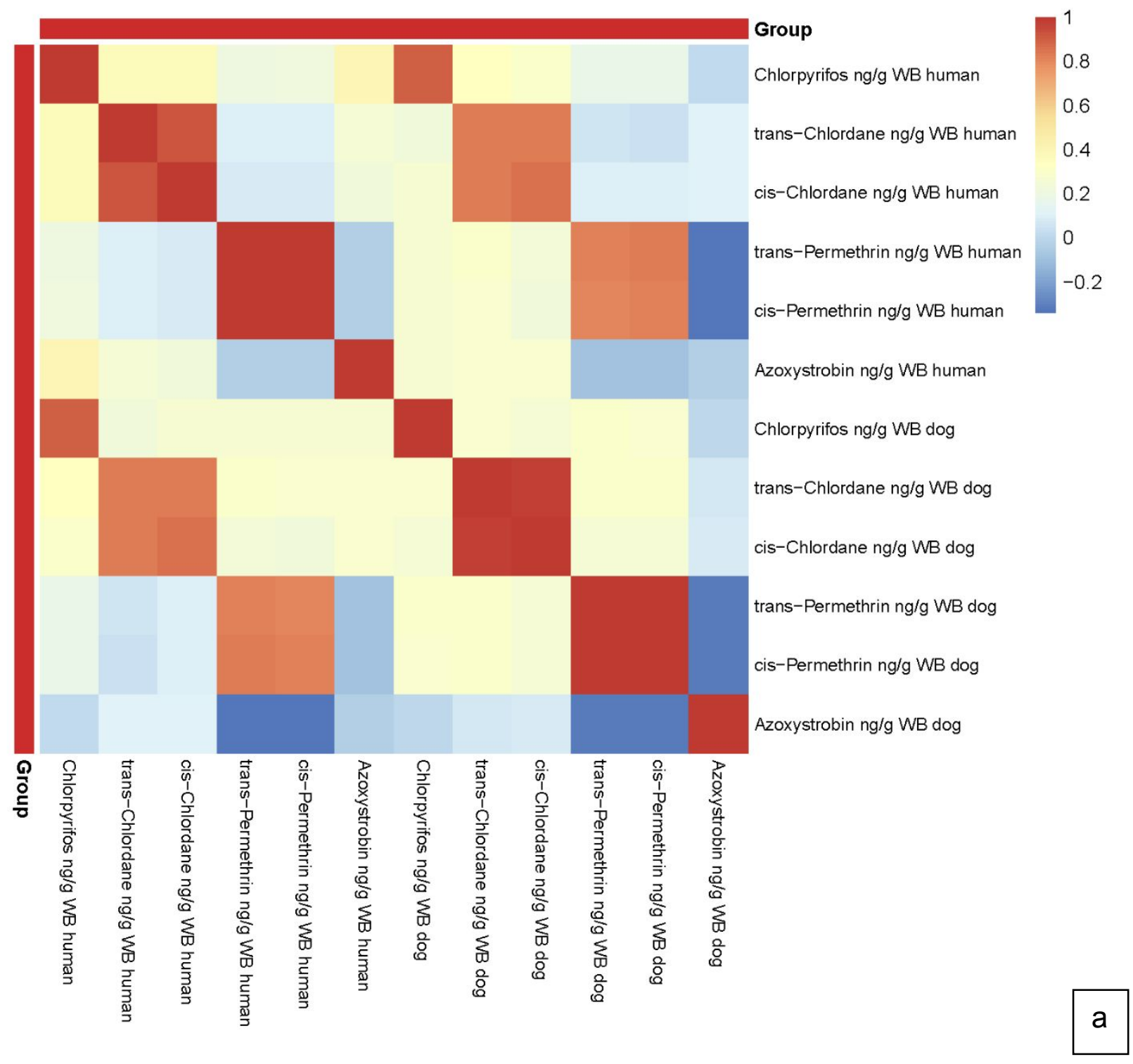




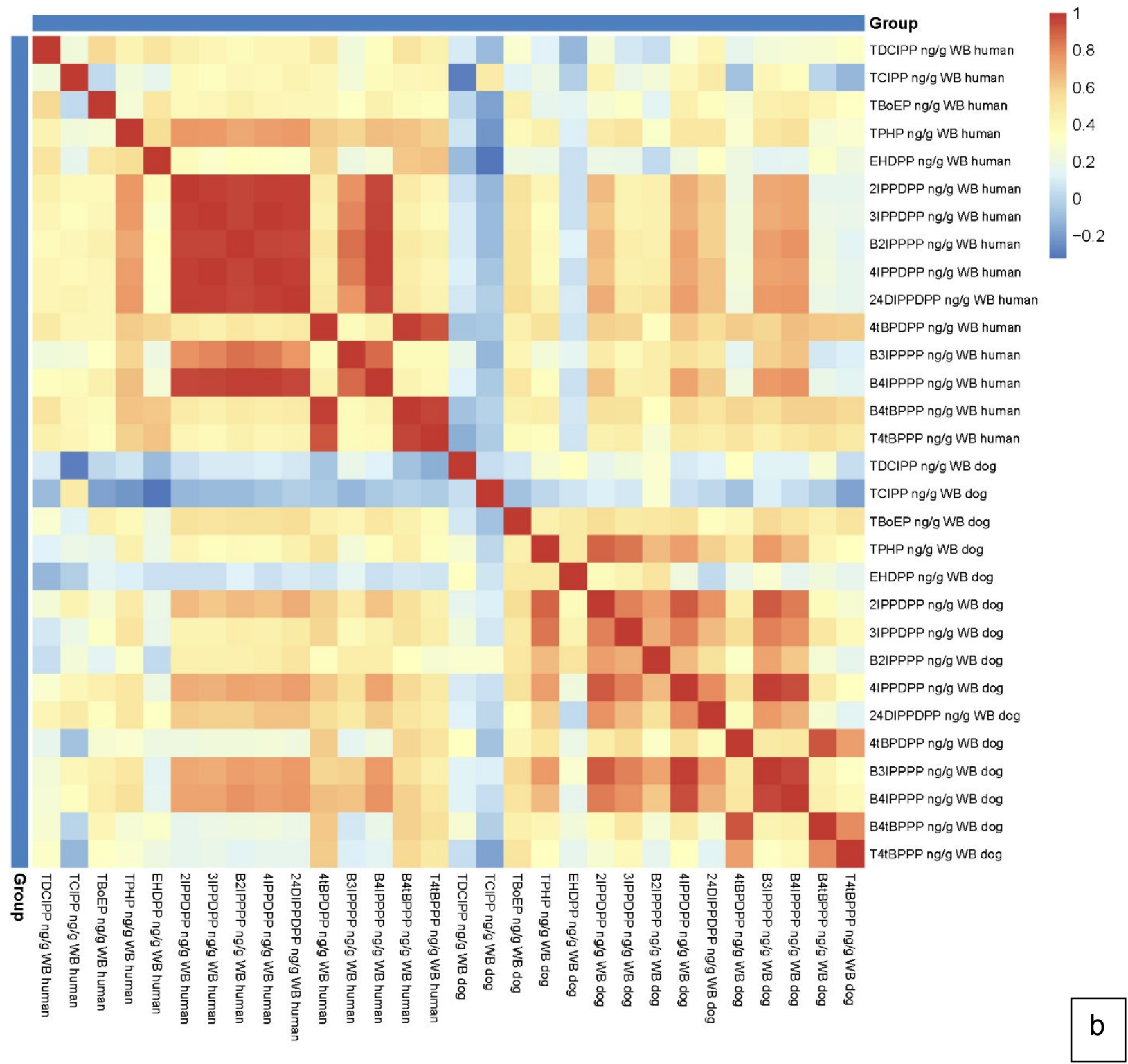




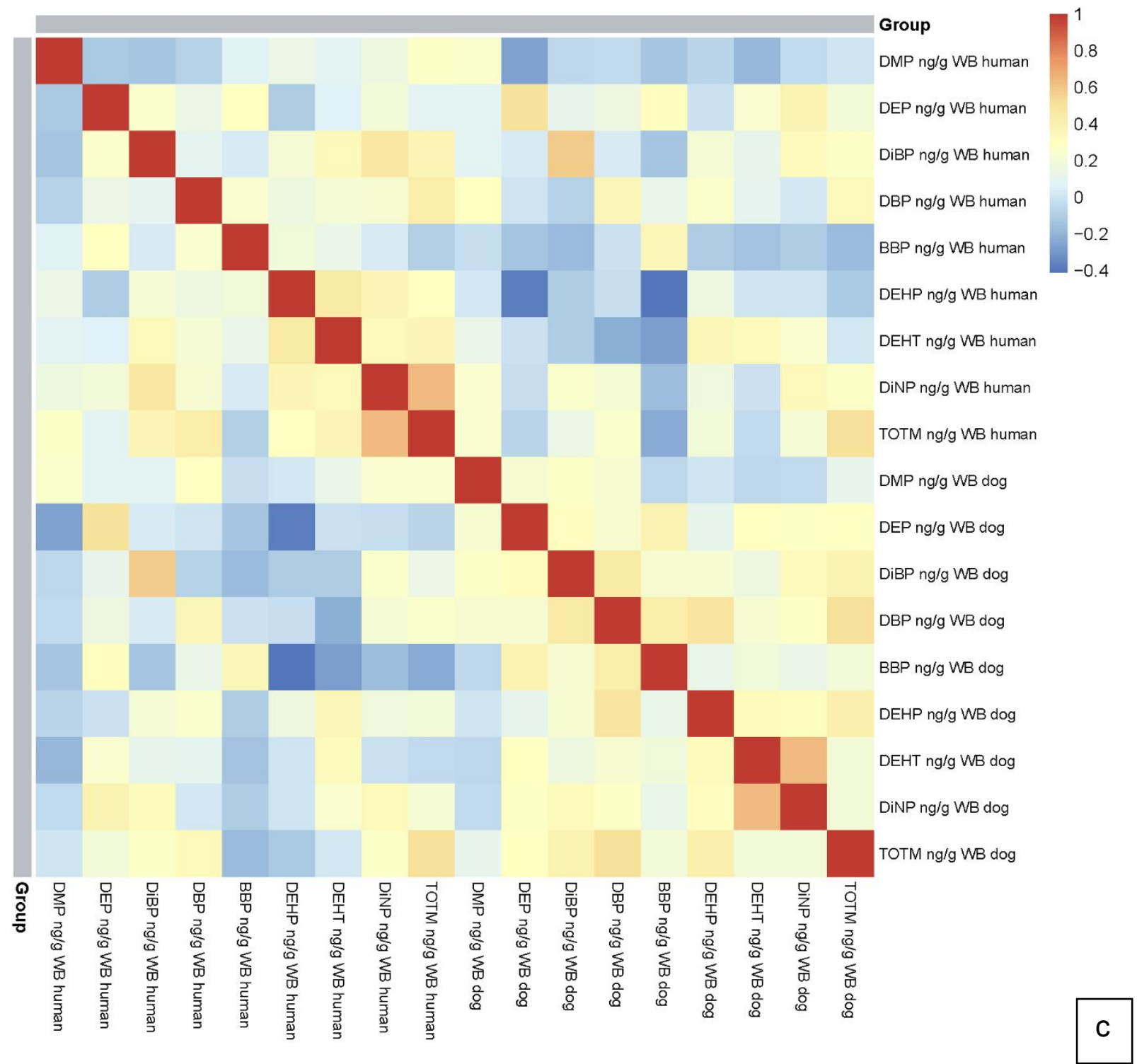




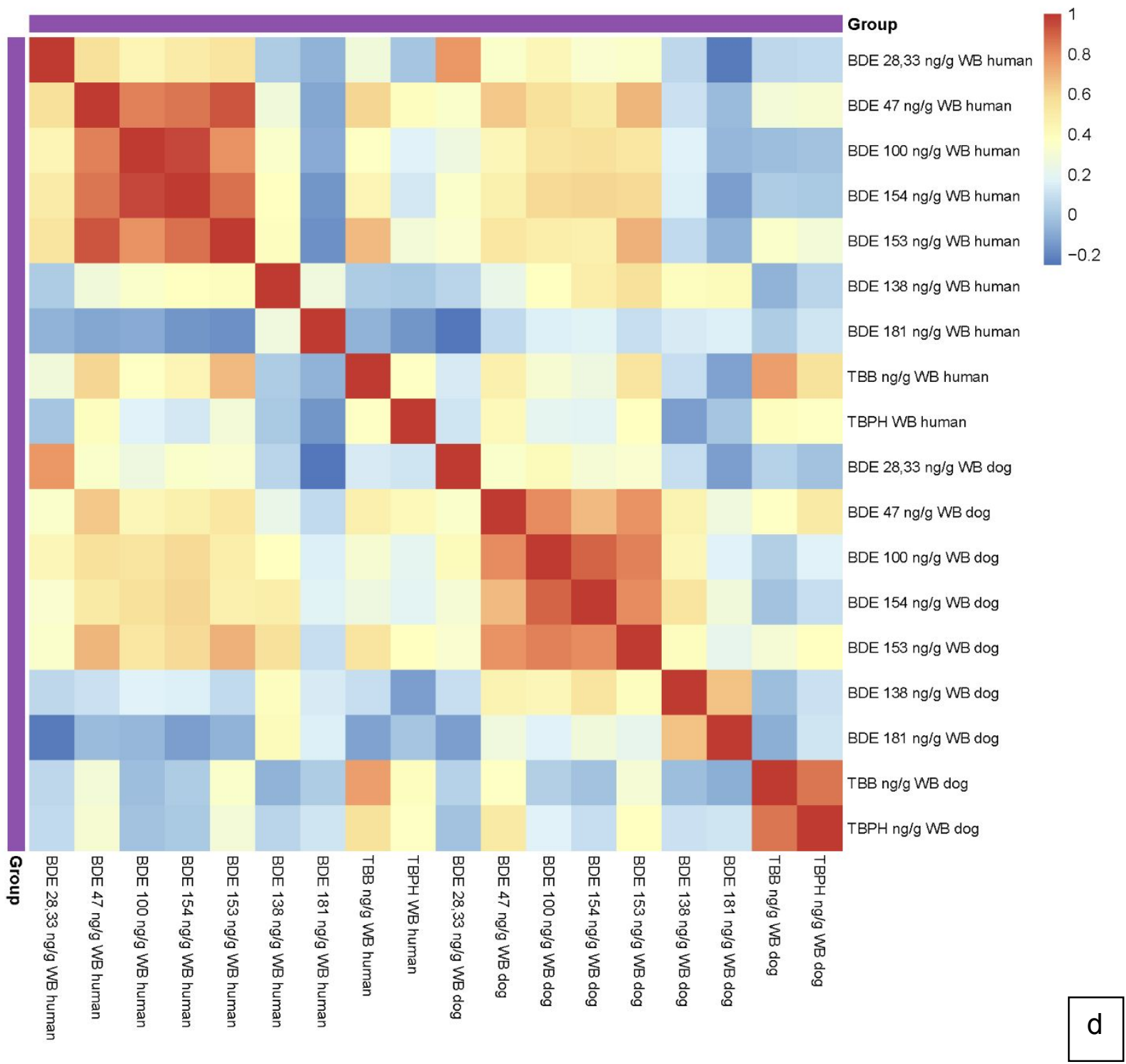




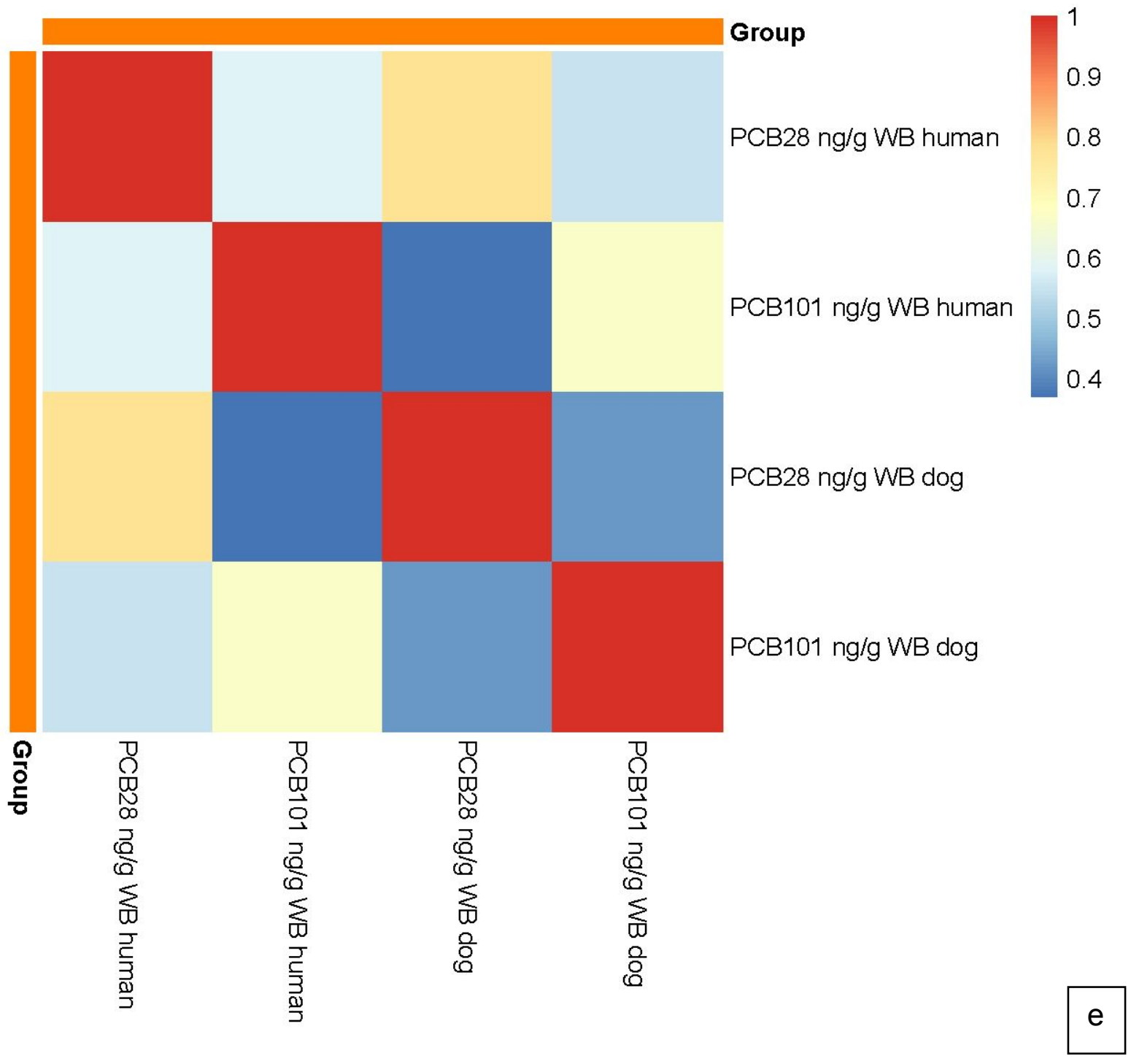

305 


\section{References only in Table S5}

56 Bergmann, A. J.; North, P. E.; Vasquez, L.; Bello, H.; Del Carmen Gastañaga Ruiz, M.; Anderson, K. A. Multi-class chemical exposure in rural Peru using silicone wristbands. $J$. Expo. Sci. Environ. Epidemiol. 2017, 27 (6), 560-568. DOI 10.1038/jes.2017.12.

57 Donald, C. E.; Scott, R. P.; Blaustein, K. L.; Halbleib, M. L.; Sarr, M.; Jepson, P. C.; Anderson, K.A. Silicone wristbands detect individuals' pesticide exposures in West Africa. R. Soc. Open Sci. 2016, 3 (8), 160433. DOI 10.1098/rsos.160433.

58 Vidi, P. A.; Anderson, K. A.; Chen, H.; Anderson, R.; Salvador-Moreno, N.; Mora, D. C.; Poutasse, C.; Laurienti, P. J.; Daniel, S. S.; Arcury, T. A. Personal samplers of bioavailable pesticides integrated with a hair follicle assay of DNA damage to assess environmental exposures and their associated risks in children. Mutat. Res. Gen. Tox. En. 2017, 822, 27-33. DOI 10.1016/j.mrgentox.2017.07.003.

59 Aerts, R.; Joly, L.; Szternfeld, P.; Tsilikas, K.; De Cremer, K.; Castelain, P.; Aerts, J-M.; Van Orshoven, J.; Somers, B.; Hendrickx, M.; Andjelkovic, M.; Van Nieuwenhuyse, A. Silicone wristband passive samplers yield highly individualized pesticide residue exposure profiles. Environ. Sci. Technol. 2018, 52 (1), 298-307. DOI 10.1021/acs.est.7b05039.

60 Manzano, C. A.; Dodder, N. G.; Hoh, E.; Morales, R. G. Patterns of personal exposure to urban pollutants using personal passive samplers and GC× GC/ToF-MS. Environ. Sci. Technol. 2019, 53, 614-624. DOI 10.1021/acs.est.8b06220. 